\title{
Manifestações musculoesqueléticas nos pacientes em programa de hemodiálise ${ }^{(*)}$
}

\section{Musculoskeletal manifestations in hemodialysis patients}

\author{
Alessandra de Sousa Braz ${ }^{(1)}$, Ângela Luzia B. P. Duarte ${ }^{(2)}$
}

\section{RESUMO}

Uma diversidade de alterações osteoarticulares tem sido descrita em pacientes em hemodiálise crônica. Objetivo: Verificar a proporção e o tipo de manifestação musculoesquelética (MME) nos pacientes em programa de hemodiálise, em três centros da região metropolitana do Recife, e relacionálas com as variáveis sexo, etnia, idade atual do paciente e, ao iniciar a diálise, tempo de tratamento dialítico. Métodos: Inicialmente, foram aplicados questionários em 197 pacientes distribuídos nos três centros no período de março de 2001 a janeiro de 2002. Após excluir 35 pacientes com diagnóstico prévio de doença reumatológica, investigou-se a presença de sinais e/ou sintomas de MME em 162 pacientes. A média de idade foi de 47,3 anos, a média da idade no início do tratamento foi de 43,8 anos, 94 pacientes (58\%) eram do sexo masculino e 120 (74,1\%), não-caucasóides. O tempo médio do tratamento dialítico foi de 44,1 meses, sendo utilizada a membrana de polissulfona em todos. Resultados: MME foram observadas em 55 (34\%) dos 162 pacientes do estudo. Destes, 38 apresentaram um único tipo de manifestação e 17 pacientes, mais de um tipo (16 apresentaram dois e um, três tipos), perfazendo um total de 73 manifestações distribuídas entre articulações (44), ossos (18), estruturas neuromusculares (seis) e periarticulares (cinco). A artralgia foi responsável por $46,6 \%$ de todas as MME, e o joelho foi a articulação mais acometida (52,9\% dos casos). A dor óssea foi a segunda queixa mais comum $(21,9 \%)$, as alterações periarticulares corresponderam a $6,8 \%$ das MME; e síndrome do túnel do carpo, deformidades ósseas e tumorações articulares ocorreram em $4,1 \%, 2,7 \%$ e $2,7 \%$, respectivamente, do total de MME. Entre os 55 pacientes, alterações articulares foram encontradas em 72,7\% deles, ósseas em 32,7\%, neuromusculares em 10,8\% e periarticulares em 9,1\% dos pacientes. Neste estudo, observou-se a relação entre o tempo médio de tratamento dialítico (59,8 meses) e o desenvolvimento de MME $(\mathrm{p}<0,001)$.

\begin{abstract}
A large diversity of ostearticular complaints has been described in patients in long-term hemodialysis. Objective: To verify the proportion and the type of musculoskeletal complaints in patients in maintenance hemodialysis, at three centers of metropolitan Recife, and to relate these complains to the following variables: sex, ethnic group, patient's current age and when the dialysis began, and dialitic treatment time. Methods: Firstly, questionnaires were applied to 197 patients, distributed at the three centers from March 2001 to January 2002. After excluding 35 patients with previous diagnosis of rheumatic disease, we investigatied the presence of musculoskeletal signs and/or symptoms in 162 patients. The average age was 47,3 years old, and 43,8 years old was the average age at the beginning of the treatment; 94 patients (58\%) were men and 120 patients (74.1\%) were not Caucasian. The average time of the dialitic treatment was 44,1 months, and polysulphone membrane was used in all of them. Results: Musculoskeletal manifestations were observed in 55 (34\%) of the patients on this research. One type of manifestation was presented by 38 of these patients, and 17 patients presented more than one type (16 presented two and 1 presented three types), making up a total of 73 manifestations, distributed among joints (44), bones (18), neuromuscular (6) and periarticular (5) structures. Arthralgia was responsible for $46.6 \%$ of all musculoskeletal complaints and the knee was the joint most injured (52.9\% of all cases). Bone pain was the second common complaint (21.9\%), periarticular changes were responsible for $6.8 \%$ of musculoskeletal manifestations, and carpal tunnel syndrome, bone deformities and joint tumours occurred in $4.1 \%, 2.7 \%$ and $2.7 \%$ of all manifestations, respectively. Among 55 patients, joint changes were found in $72.7 \%$ of them, bone changes in $32.7 \%$, neuromuscular complaints in $10.8 \%$ and periarticular in $9.1 \%$ of the patients. The relation between the dialitic treatment time (59,8 months) and the development of the musculoskeletal manifestations $(p<0.001)$ was observed in this research.
\end{abstract}

\footnotetext{
* Trabalho realizado no Departamento de Medicina Clínica do Centro de Ciências de Saúde da Universidade Federal de Pernambuco (UFPE). Recebido em 31/7/2002. Aprovado, após revisão, em 13/2/2003.

1. Mestre em Medicina Interna (área de concentração em Reumatologia) pela UFPE.

2. Professora Titular da UFPE e Chefe da Unidade Funcional de Reumatologia do Hospital das Clínicas da UFPE.
}

Endereşo para correspondência: Alessandra Braz. Rua Dr. Francisco de Assis C. Dantas, 270, ap. 403, CEP 58.036-399, Bessa, João Pessoa, PB, e-mail: alesbraz@bol.com.br 
Conclusões: a partir destes dados, conclui-se que a proporção de MME nos nossos pacientes foi de 34\%; as manifestações articulares foram as mais observadas e a artralgia foi a queixa mais comum. No nosso estudo, com exceção do tempo de tratamento dialítico, as demais variáveis não mostraram associação com o aparecimento de MME.

Palavras-chave: Hemodiálise, manifestações musculoesqueléticas, tempo de tratamento dialítico.

\section{INTRODUÇÃO}

A hemodiálise intermitente crônica começou a ser realizada no ano de 1960, na Universidade de Washington, quando, com o transplante renal, aumentou-se consideravelmente a expectativa de vida de muitos pacientes com insuficiência renal crônica (IRC) ${ }^{(1,2)}$.

A hemodiálise (HD) é a modalidade de tratamento dialítico mais utilizada. Atualmente, entre 30.000 e 35.000 pacientes com IRC são tratados com diálise crônica no Brasil, e estima-se que no mínimo 80 novos pacientes $/ 10^{6}$ habitantes/ano passem a precisar de tal tratamento ${ }^{(3)}$.

Durante a realização da HD, podem ocorrer complicações agudas como arritmias, hipotensão arterial, náuseas, cãibras, problemas no acesso vascular (tromboses e/ou infecções), e, quando realizada em regime intermitente prolongado, pode-se acompanhar de manifestações musculoesqueléticas (MME) em até 69\% dos $\operatorname{casos}^{(4)}$.

Como o rim participa do metabolismo do cálcio, do fósforo, do hormônio paratireóideo (PTH) e da vitamina $\mathrm{D}$, uma conseqüência esperada é o aparecimento de alterações ósseas e minerais à medida que ocorre a destruição do parênquima renal ${ }^{(5)}$. O surgimento dessas alterações varia de acordo com cada paciente, devendo-se, provavelmente, a fatores como idade, duração da insuficiência renal, dieta, terapêutica medicamentosa utilizada e duração do tratamento dialítico ${ }^{(6)}$.

Uma das mais graves manifestações musculoesqueléticas observadas nos pacientes em fases avançadas da insuficiência renal é a osteodistrofia, que se refere a um complexo de alterações do metabolismo mineral envolvendo o esqueleto e outros órgãos ${ }^{(7,8)}$. Tais alterações são incomuns no paciente não submetido à diálise, mas o tratamento dialítico prolongado propiciou o seu aparecimento com maior freqüência ${ }^{(9)}$.

Embora a osteodistrofia renal (OR) esteja freqüentemente presente nos pacientes em hemodiálise, ela explica queixas artríticas em apenas uma minoria deles ${ }^{(10)}$.
Conclusions: According to this information, we can conclude that the proportion of musculoskeletal complaints on our patients was 34\%; the joint problems were the most frequently observed; and arthralgia was the most common complaint. Except for the dialitic treatment time, all the other variables were not associated with the musculoskeletal manifestations.

Keywords: hemodialysis, musculoskeletal manifestations, dialitic treatment time.

Uma variedade de anormalidades afetando as articulações e áreas periarticulares têm sido descritas em pacientes urêmicos recebendo hemodiálise cronicamente. As mais freqüentemente observadas incluem artrites induzidas por cristais $^{(1,11)}$, infecções piogênicas de articulações e/ou os$\operatorname{sos}^{(12-14)}$, artropatia crônica amilóide ${ }^{(15-18)}$, espondiloartropatia destrutiva ${ }^{(19-21)}$, hemartrose recorrente ${ }^{(4,22)}$, bursite olecraniana ${ }^{(23)}$, artropatia por alumínio ${ }^{(24-26)}$, calcificação de partes moles ${ }^{(2,9)}$ e necrose isquêmica óssea ${ }^{(6,27)}$, entre outras.

A escassez de estudos que analisem doenças musculoesqueléticas em pacientes em programa de hemodiálise na literatura brasileira nos motivou a estudar a freqüência dessas manifestações nos pacientes em hemodiálise em três centros da região metropolitana de Recife.Sabendo-se que tais manifestações podem ocorrer em até 69\% desses pacientes, em especial naqueles em tratamento prolongado, da importância dessa complicação na vida dos pacientes em diálise e da alteração na sua qualidade de vida em decorrência da incapacidade funcional dolorosa por elas determinada, o reconhecimento precoce de tais manifestações poderá ser capaz de aliviar a sintomatologia e melhorar a qualidade de vida desses pacientes.

Objetivando verificar a proporção de manifestações musculoesqueléticas nos pacientes em programa de hemodiálise na região metropolitana da cidade de Recife e relacionálas com as variáveis sexo, etnia, idade atual do paciente e, ao iniciar a diálise, tempo do tratamento dialítico, os autores investigaram a presença de sinais e/ou sintomas musculoesqueléticos em 162 pacientes em hemodiálise crônica.

\section{PACIENTES E MÉTODOS}

Realizou-se um estudo descritivo observacional, do tipo série de casos, de base hospitalar/serviços de saúde.

Participaram do estudo 162 pacientes em programa de hemodiálise nos seguintes centros da região metropolitana de Recife: Nefroclínica (47 pacientes), Serviços Nefrológicos Ltda. - Prórim (49 pacientes) e Unirim (66 pacientes). 
Inicialmente, foi selecionada uma amostra de $197 \mathrm{pa}-$ cientes a partir de uma população de 481 indivíduos em programa de HD nos três centros referidos, no período de março de 2001 a janeiro de 2002. Do total de 561 pacientes em tratamento naqueles centros no início do estudo, 80 não foram incluídos nesta seleção inicial por apresentarem teste sorológico positivo para hepatite por vírus B e/ ou C (24 na Nefroclínica; 17 na Prórim e 39 na Unirim).

O cálculo do tamanho da amostra foi determinado com o objetivo de estimar a proporção de pacientes em programa de hemodiálise com manifestações musculoesqueléticas. Admitindo-se um erro máximo de estimação de 5\%, ao nível de confiança de 95\%, e uma prevalência esperada de $69 \%$, conforme observado por Brown e Gower $(1982)^{(4)}$, obteve-se um tamanho de amostra igual a 197 pacientes.

A partir de um total de 481 pacientes em tratamento nas três clínicas, foi feito um cálculo proporcional do número de pacientes de cada clínica a ser sorteado. Na Nefroclínica, com 152 pacientes, foram sorteados 62; na Prórim, com 143, foram sorteados 59; e na Unirim, com 186 pacientes, foram sorteados 76 .

Os participantes foram selecionados pelo método de amostragem aleatória sistemática: a cada dois pacientes, um era incluído. O sorteio dos pacientes foi realizado utilizando as folhas de controle dos turnos de hemodiálise de cada clínica contendo os primeiros nomes dos pacientes.

Os dados foram obtidos por meio da aplicação de questionários, elaborados especificamente para este estudo, contendo identificação do paciente, etiologia da insuficiência renal, tempo e tipo de tratamento dialítico utilizado, terapia medicamentosa concomitante, anamnese e exame fisico reumatológico realizados por um único pesquisador.

Após a aplicação do questionário, foram excluídos 35 pacientes que apresentavam doença reumatológica previamente confirmada, ou que referissem qualquer tipo de manifestação osteoarticular antes do início do tratamento dialítico (episódio de artrite de etiologia indeterminada, bursopatias, dores difusas nos ossos, entre outros, não devidamente investigados ou não diagnosticados), visando evitar que tais alterações funcionassem como um "fator de confusão" na análise dos resultados. Permaneceram 162 pacientes no estudo.

Foi considerado como portador de dor óssea secundária ao tratamento dialítico aquele paciente que apresentasse dor difusa, progressiva, em geral agravada por aumento do peso corpóreo ou da postura do paciente, localizada nos membros, obrigatoriamente relacionada com o início do tratamento dialítico.
De modo semelhante, fizeram parte do estudo os pacientes que referiram dores articulares difusas, no repouso, com rigidez articular e/ou limitação dos movimentos, podendo estar associadas com aumento do peso corporal. Foram incluídas como portadores de artralgia as articulações isentas de outras queixas investigadas no trabalho; os pacientes que apresentaram artrite, dor óssea ou bursopatias, entre outras, não foram considerados quanto à presença de artralgia.

Dos 162 pacientes que fizeram parte do estudo, não foram incluídos como portadores de MME aqueles que, apesar de não apresentarem queixas antes do início da diálise, referiam dores ao longo da coluna ou dores protocinéticas e crepitações grosseiras, especialmente unilaterais, nas articulações. Os primeiros, por não existirem parâmetros clínicos eficazes para descartar a participação de osteoartrose ou do fator postural em sua etiologia, e os segundos por se tratarem de queixas características de osteoartrose. Tal conduta foi adotada pelos autores visando reduzir a participação de outros fatores etiológicos capazes de prejudicar os nossos resultados.

Todos os participantes assinaram o termo de consentimento livre e esclarecido para a participação no trabalho. Nenhum paciente se recusou a participar do estudo.

A análise estatística foi realizada utilizando-se o teste Quiquadrado para as variáveis qualitativas e o teste T de Student para verificar diferença de médias entre as variáveis do estudo.

\section{RESULTADOS}

A idade dos pacientes do estudo variou de 18 a 91 anos (média de 47,3 anos e mediana de 47,00); 94 (58\%) eram do sexo masculino e 68 (42\%), do feminino. Com relação à etnia, 42 pacientes $(25,9 \%)$ eram caucasóides e 120 (74,1\%) não-caucasóides.

Entre as doenças possivelmente associadas com o desenvolvimento da insuficiência renal, as mais freqüientes foram hipertensão arterial sistêmica (HAS), presente em 56,8\% dos pacientes, diabetes mellitus, em $14,2 \%$ dos pacientes, e glomerulonefrite crônica (GNC), em 12,3\% (Tabela 1).

O tempo médio de duração do tratamento dialítico foi de 44,1 meses. Todos os pacientes faziam hemodiálise com a membrana de polissulfona, durante três vezes por semana e com duração de 12 horas semanais. O consumo médio de comprimidos de carbonato de cálcio e de vitamina D (forma ativa) pelos 162 pacientes foi de 6 e 1,12, respectivamente. 
TABELA 1

POSSÍVEIS CAUSAS DE INSUFICIÊNCIA RENAL NOS 162 PACIENTES (MAIS DE UMA CAUSA PODE ESTAR PRESENTE)

\begin{tabular}{ll}
\hline Doença & Freqüência de pacientes $(\%)$ \\
\hline Hipertensão arterial sistêmica & $92(56,8)$ \\
Diabetes mellitus & $23(14,2)$ \\
Glomerulonefrite crônica & $20(12,3)$ \\
Rins policísticos & $10(6,2)$ \\
Indeterminadas & $18(11,1)$ \\
Outras & $22(13,6)$ \\
\hline
\end{tabular}

\section{MANIFESTAÇÕES MUSCULOESQUELÉTICAS}

As manifestações musculoesqueléticas foram observadas em 55 (34\%) dos 162 pacientes do estudo. A distribuição dos pacientes com manifestações musculoesqueléticas entre as clínicas ocorreu da seguinte forma: 14 na Prórim, 21 na Unirim e 20 na Nefroclínica.

Dos 55 pacientes que apresentaram algum tipo de manifestação musculoesquelética, 38 pacientes apresentaram um único tipo de manifestação e 17 pacientes apresentaram mais de um (16 apresentaram dois e um apresentou três tipos), perfazendo um total de 73 manifestações distribuídas entre articulações, ossos, estruturas periarticulares e neuromusculares. Dessas, 44 foram articulares; 18, ósseas; 5 , periarticulares e 6 , neuromusculares.

Entre as manifestações articulares, a artralgia representou $46,57 \%$ do total de MME, a artrite, $11 \%$, e as tumorações, $2,7 \%$. Com relação às alterações ósseas, a dor óssea representou $21,9 \%$ de todas as manifestações referidas, e as deformidades, 2,7\%. Mialgia e síndrome do túnel do carpo ocorreram com a mesma freqüência: $4,1 \%$ do total, cada, e as alterações periarticulares corresponderam a $6,8 \%$ de todas as manifestações encontradas (Tabela 2).

\section{MANIFESTAÇÕES ARTICULARES}

Entre as MME apresentadas pelos 55 pacientes, o envolvimento articular ocorreu em 40 pacientes $(72,7 \%)$. Dois tipos de manifestações articulares (artralgia e artrite) foram observados em três deles, e artralgia e tumoração articular, em um. Portanto, foram encontrados 44 tipos de manifestações articulares nos 40 pacientes: 34 casos de artralgia, oito de artrite e dois de tumorações articulares.

A artralgia foi observada em $61,8 \%$ dos 55 pacientes do estudo, e o joelho foi a articulação mais acometida por ela $(52,9 \%$ dos casos), seguida pelos ombros $(35,3 \%)$
(Tabela 3). Entre os pacientes com artralgia, 20 apresentaram apenas artralgia, 8 tiveram dor óssea associada (em outra área do corpo) e 3, artralgia em uma articulação e artrite em outra. Os três pacientes restantes apresentaram artralgia e STC, artralgia e tumoração na mão e artralgia e bursite em áreas distintas.

A artrite foi demonstrada em 8 pacientes (14,5\% dos pacientes do estudo e 18,1\% das manifestações articulares): em 3 nos tornozelos e nos punhos; em 2 nas interfalangeanas; e em 1 no joelho, sendo que um dos 8 apresentou artrite em interfalangeanas e punho simultaneamente. Três pacientes apresentaram artrite como única manifestação, e os demais foram associados com artralgia (3 pacientes), bursite (1 paciente) e dor óssea (1 paciente), em locais diferentes.

Tumorações articulares foram observadas em dois pacientes (3,6\% dos pacientes do estudo e 4,5\% das manifestações articulares): em um, de tamanho gigante, na cintura escapular bilateralmente, e no outro na articulação carpometacarpiana do quinto dedo da mão direita, medindo aproximadamente $3 \mathrm{~cm}$ no seu maior diâmetro.

TABELA 2

FREQÜÊNCIA DOS 73 SINAIS E SINTOMAS MUSCULOESQUELÉTICOS APRESENTADOS PELOS PACIENTES DO ESTUDO

\begin{tabular}{ll}
\hline Sinais e sintomas & Freqüência $(\%)$ \\
\hline Artralgia & $34(46,57)$ \\
\hline Dor óssea & $16(21,91)$ \\
\hline Artrite & $8(11)$ \\
Alterações periarticulares & $5(6,84)$ \\
Mialgia & $3(4,10)$ \\
\hline Síndrome do túnel do carpo & $3(4,10)$ \\
\hline Tumorações articulares & $2(2,74)$ \\
Deformidades ósseas & $2(2,74)$ \\
\hline
\end{tabular}

TABELA 3

ACOMETIMENTO ARTICULAR NOS 34 PACIENTES COM ARTRALGiA (MAIS DE UMA ARTICULAÇÃO PODE ESTAR ACOMETIDA)

\begin{tabular}{ll} 
Articulações & Freqüência (\%) de articulações \\
\hline Joelhos & $18(52,9)$ \\
\hline Ombros & $12(35,3)$ \\
\hline Tornozelos & $6(17,6)$ \\
\hline Interfalangeanas & $5(14,7)$ \\
Cotovelos & $5(14,7)$ \\
Punhos & $4(11,7)$ \\
\hline
\end{tabular}




\section{MANIFESTAÇÕES NO TECIDO ÓSSEO}

Do total dos 55 pacientes, 18 (32,7\%) tiveram sintomatologia óssea, $16(29,1 \%)$ apresentando dor óssea e $2(3,6 \%)$, deformidades.

Entre os pacientes com dor óssea, 4 referiam dor difusa (membros superiores e inferiores); 10, dores nos ossos das pernas; 1, nos braços e 1, nas costelas. Houve associação com outra manifestação em 9 pacientes.

As deformidades ósseas foram observadas em apenas duas pacientes, ambas com distorção da caixa torácica e cifoescoliose. Uma delas apresentava tenossinovite dos tendões flexores dos dedos, e a outra, deformidade nos ossos da face (mandíbula esquerda) e do crânio (osso frontal à esquerda).

\section{MANIFESTAÇÕES PERIARTICULARES}

Entre os cinco pacientes que apresentaram manifestações periarticulares $(9,1 \%)$, um apresentava bursite olecraniana unilateral esquerda e outro, tenossinovite crônica bilateral dos dedos.

\section{MANIFESTAÇÕES NEUROMUSCULARES}

Em relação às manifestações neuromusculares, a mialgia foi demonstrada em três pacientes $(5,4 \%)$, sendo demonstrados dois casos de mialgia localizada (panturrilhas) e um de mialgia difusa. A STC também foi diagnosticada com a mesma frequiência e, em dois pacientes, ocorreu bilateralmente.

Com relação às variáveis estudadas, quando avaliamos o sexo, a etnia, a idade atual do paciente e ao iniciar a hemodiálise, após aplicar o teste Qui-quadrado, não foi observada diferença estatisticamente significativa entre tais variáveis e a presença de manifestações musculoesqueléticas. Não sendo constatada, portanto, nenhuma associação entre estas variáveis e o desenvolvimento de MME nesse grupo de pacientes.

$\mathrm{Na}$ Tabela 4, observa-se diferença estatisticamente significativa entre as médias de tempo de tratamento dialítico, em meses, e a presença ou ausência de manifestações musculoesqueléticas (teste $\mathrm{T}$ de Student para amostras independentes $-\mathrm{p}<0,001)$. Os pacientes que apresentaram MME tiveram maior tempo médio de tratamento $(59,8$ meses) do que os que não referiram sinais e/ou sintomas musculoesqueléticos (36,9 meses), ficando, portanto, comprovada a associação entre o tempo de tratamento dialítico e o desenvolvimento de MME nesses pacientes.

As principais características dos 162 pacientes do estudo com e sem MME são mostradas na Tabela 5. Aplicou-se o teste T-Student para amostras independentes nos grupos com e sem alterações nas variáveis: idade atual e ao iniciar o tratamento dialítico, tempo de tratamento dialítico e uso do carbonato de cálcio e vitamina D na forma ativa.

\section{DISCUSSÃO}

O advento da diálise no tratamento dos pacientes com doença renal em estágio terminal permitiu um importante aumento na sobrevida desses pacientes e, conseqüentemente, aumentou a freqüência e a gravidade do aparecimento de doença osteoarticular relacionada com o tratamento dialítico prolongado ${ }^{(8)}$.

Têm sido descritas muitas manifestações musculoesqueléticas nos pacientes em hemodiálise. As complicações articulares representam o principal fator limitante para seu prognóstico em virtude da incapacidade funcional dolorosa por elas provocada ${ }^{(13)}$.

\section{TABELA 4}

DisTRIBUIÇÃO DOS PACIENTES SEGUNDO A MÉDIA DE TEMPO DE TRATAMENTO DiALÍTICO (TTD) EM MESES E A PRESENÇA DE MANIFESTAÇÕES MUSCULOESQUELÉTICAS APÓS HEMODIÁLISE CRÔNICA

\begin{tabular}{lcccccc}
\hline $\begin{array}{l}\text { MME } \\
\text { após HD }\end{array}$ & $\mathbf{n}$ & $\begin{array}{c}\text { Média de } \\
\text { TTD (meses) }\end{array}$ & DP & T & gl & p \\
\hline Presença & 55 & 59,80 & 43,74 & 3,42 & 84,72 & 0,001 \\
Ausência & 107 & 36,99 & 32,09 & & & \\
\hline
\end{tabular}

\section{TABELA 5}

Principais CARACTERÍSTICAS DOS 162 PACIENTES DO ESTUDO COM E SEM MANIFESTAÇÕES MUSCULOESQUELÉTICAS APÓS HEMODIÁLISE DE MANUTENÇÃO

\begin{tabular}{lcc}
\hline $\begin{array}{l}\text { Características } \\
\text { dos pacientes }\end{array}$ & $\begin{array}{c}\text { Pacientes } \\
\text { com MME }\end{array}$ & $\begin{array}{c}\text { Pacientes } \\
\text { sem MME }\end{array}$ \\
\hline Número de pacientes & 55 & 107 \\
\hline Homens/mulheres & $29 / 26$ & $65 / 42$ \\
Caucasóides/não-caucasóides & $11 / 44$ & $76 / 31$ \\
\hline Idade atual dos pacientes (média) & 48,35 & 46,80 \\
Idade no início do tratamento (média) & 43,60 & 44,23 \\
\hline Tempo de tratamento dialítico & 59,80 & 36,99 \\
$\quad$ em meses (média) & & \\
Tipo de membrana utilizada & polissulfona & polissulfona \\
Uso de carbonato de cálcio (média) & 5,62 & 6,23 \\
Uso de vitamina D ativa (média) & 1,29 & 1,04 \\
\hline
\end{tabular}


A freqüência de manifestações musculoesqueléticas nesse grupo de pacientes é variável, e no estudo feito por Brown e Gower ${ }^{(4)}$ alterações articulares foram demonstradas em $69 \%$ dos pacientes.

No nosso estudo, a proporção de MME nos pacientes em HD foi de 34\%. Goldstein et al. ${ }^{(28)}$, entretanto, verificaram um elevado porcentual de manifestações musculoesqueléticas (73\%) nos seus pacientes, possivelmente por causa do número reduzido da sua amostra (11) e pelo fato de que todos estavam em programa de HD há mais de dez anos.

Entre as MME mais freqüentemente referidas no estudo aqui apresentado, as articulares ocorreram em $72,7 \%$ dos pacientes, de modo semelhante ao observado nos estudos de Brown e Gower ${ }^{(4)}$ e Hardouin et al. ${ }^{(29)}$ com $69 \%$ e $72 \%$, respectivamente.

Menerey et al. ${ }^{(10)}$, em estudo prospectivo com 28 pacientes, verificaram queixas artríticas com freqüência nos pacientes em diálise, sendo a artropatia responsável por $61 \%$ das queixas e a osteodistrofia renal (OR) por 17\% das alterações articulares. Segundo os autores, a OR explicou queixas artríticas em uma minoria desses pacientes.

Chou et al. ${ }^{(30)}$ encontraram artralgias em 19,6\% dos seus pacientes, 3\% de poliartrites, e derrame articular de causa indeterminada em 4\%. Nesse estudo, em 28 pacientes, as alterações foram demonstradas radiograficamente e a correlação entre a artralgia e os achados radiográficos foram pobres. No nosso estudo, a artralgia ocorreu em 61,8\% dos pacientes e a artrite, em 14,5\%. A comparação com os resultados deste estudo é difícil, uma vez que aqueles autores não excluíram os pacientes com manifestações reumatológicas prévias e utilizaram métodos diagnósticos complementares.

Observou-se que a artralgia, responsável por $77,2 \%$ das alterações articulares apresentadas, ocorreu predominantemente nos joelhos (52,9\% dos pacientes) e ombros $(35,3 \%)$, acompanhada do acometimento de tornozelos $(17,6 \%)$, interfalangeanas $(14,7 \%)$, cotovelos $(14,7 \%)$ e punhos $(11,7 \%)$. No estudo de Brown e Gower ${ }^{(4)}$, a dor articular ocorreu com mais freqüência nas mãos $(64,4 \%$ dos pacientes), seguida pelos pés $(59,3 \%)$, joelhos $(54,2 \%)$, ombros $(50,8 \%)$ e cotovelos $(35,6 \%)$, em um total de 59 pacientes com artralgia.

Detectou-se artrite em oito pacientes, mas não foi possível determinar sua etiologia; pois, sendo de um estudo observacional, não foi realizada a punção articular diagnóstica. Dois pacientes que apresentaram artrite faziam HD há mais de cinco anos, e as características clínicas (exames fisicos geral e articular) afastaram o possível diagnóstico de artrite piogênica e/ou metabólica.

Entre os pacientes do nosso estudo, um, com 36 anos e em hemodiálise há cinco, apresentava aumento "tumoral" bilateral de grandes proporções, sobretudo no ombro direito, de consistência fibroelástica, além de tumoração axilar que impedia a abdução dos membros superiores. Por ocasião do desenvolvimento das alterações, o paciente estava há um ano e meio sem usar carbonato de cálcio e/ou calcitriol, com sérios problemas adaptativos na HD.

Depósitos de proteína amilóide em topografia do manguito rotador do ombro, podendo limitar sua mobilidade e tornarem-se grandes e visíveis ao exame físico, foram descritos por Lazarus, Denker e Owen Jr. ${ }^{(17)}$. Com base nesses achados, mesmo na impossibilidade de realizar métodos complementares de diagnóstico, acreditamos tratarse de um caso de artropatia por depósitos amilóides.

Entre as manifestações periarticulares, a bursite olecraniana aparece em até 3\% dos pacientes em HD, em geral no membro que contém a fistula arteriovenosa, e sua patogênese associa-se a microtraumas no cotovelo durante as sessões de diálise ${ }^{(23)}$. Na nossa casuística, um paciente $(1,8 \%)$ de 61 anos apresentava quadro crônico-agudizado de bursite olecraniana unilateral esquerda, com três anos de evolução, no membro que continha a fístula.

A dor óssea foi a segunda MME mais freqüente (16 pacientes), ocorrendo predominantemente nos membros inferiores, de forma difusa, relacionada com as alterações do peso corporal e aparecendo no repouso, em concordância com a literatura, que atribui tal sintomatologia à $\mathrm{OR}^{(8,9)}$.

As deformidades ósseas, características de osteodistrofia renal $^{(9)}$, foram detectadas em duas pacientes $(3,6 \%$ dos pacientes). Ambas, adultas no início da diálise, estavam em tratamento por muitos anos: uma, em hemodiálise há nove anos, apresentava cifoescoliose e aumento dos ossos do crânio e da face; a outra, há doze anos em tratamento, desenvolveu cifoescoliose, diminuição importante da estatura e deformidades nas mãos.

Em 1975, Massry et al. ${ }^{(6)}$ afirmaram que o desenvolvimento de alterações osteoarticulares em pacientes urêmicos era dependente de fatores individuais e, provavelmente, tinha relação com idade, duração da insuficiência renal, dieta, terapêutica medicamentosa utilizada e duração do tratamento dialítico. Desde então, inúmeros trabalhos vêm tentando detectar os fatores associados com o desenvolvimento de MME nesses pacientes.

Em relação às variáveis estudadas, não foi observada associação entre sexo, etnia, idade atual dos pacientes, idade ao iniciar tratamento dialítico e o aparecimento de MME, 
mas verificamos uma relação entre os pacientes com tais manifestações e o tempo de tratamento dialítico.

Concordante com os nossos resultados, Van Ypersele de Strihou et al. ${ }^{(31)}$ e Sethi et al. ${ }^{(32)}$ não verificaram associação positiva entre o sexo e o desenvolvimento de MME.

Gaucher et al. ${ }^{(33)}$, Gueerlings et al. ${ }^{(34)}$ e Kessler et al. ${ }^{\left({ }^{(35)}\right.}$ observaram relação entre a idade atual dos pacientes e a ocorrência de MME, diferentemente dos nossos resultados e dos de Sethi et al. ${ }^{(32)}$.

De modo semelhante, a idade do paciente ao iniciar o tratamento dialítico não mostrou associação com o aparecimento de MME nos nossos pacientes, contrariando os achados de Schiffl et al. ${ }^{(36)}$.

O nosso estudo demonstrou uma associação estatisticamente significativa entre o tempo do tratamento dialítico e o surgimento de MME ( $p<0,001)$, ou seja, as manifestações musculoesqueléticas ocorreram nos pacientes com maior tempo de tratamento dialítico (59,8 meses ou 5 anos) quando comparados com os que não apresentaram tais manifestações (36,9 meses). Esses aspectos também foram observados nos estudos de Brown e Gower ${ }^{(4)}$, Rubin et al. ${ }^{(37)}$, Goldstein et al. ${ }^{(28)}$, Hardouin et al. ${ }^{(29)}$ e Sethi et al. ${ }^{(32)}$.

A freqüência de MME no estudo de Hardouin et al. ${ }^{(29)}$ parece ter sido maior do que a relatada por Brown e Gower ${ }^{(4)}$ e por Rubin et al. ${ }^{(37)}$, mas nestes últimos o tempo médio de tratamento dialítico foi menor $(63$ e 69 meses, respectivamente), e no estudo de Brown e Gower ${ }^{(4)}$ os pacientes que tiveram maior grau de comprometimento funcional foram aqueles que tinham feito diálise por um maior período de tempo (73 meses), quando comparado com os pacientes que não apresentaram sintomas (48 meses).

Halter et al. ${ }^{(38)}$, entretanto, não observaram nenhuma relação entre o tempo de tratamento dialítico e o aparecimento de artropatia amilóide, e Gaucher et al. ${ }^{(33)}$ encontraram artropatia associada à diálise em 57 dos 124 pacientes do seu estudo e concluíram que, independentemente da duração média do tratamento dialítico entre os pacientes com ou sem manifestações articulares, os pacientes com alterações foram significativamente mais velhos que os sem alterações $(\mathrm{p}<0,001)$.

Rubin et al. ${ }^{(37)}$ demonstraram associação entre o período de tratamento dialítico e o desenvolvimento de artropatia erosiva, sendo a duração do tratamento significativamente maior nos pacientes com artropatia erosiva $(69,6$ meses) do que nos pacientes com sintomas articulares, mas sem artropatia erosiva (54,7 meses).

A duração média do tratamento dialítico (em anos) de dois dos pacientes que apresentaram síndrome do túnel do carpo (STC) bilateral (tipicamente associado com artropatia associada à diálise - AAD) foi de dez anos, e na paciente que desenvolveu a tenossinovite dos tendões flexores dos dedos o período foi de 12 anos. Deposição da proteína amilóide nas bainhas dos tendões flexores dos dedos, podendo resultar em deformidade permanente e rupturas tendíneas foi descrita por Wendling e Guidet ${ }^{(23)}$ e Lazarus, Denker e Owen Jr. ${ }^{(17)}$.

Com relação à artralgia, dos 34 pacientes com a queixa, quase a metade (16) estavam em tratamento há mais de cinco anos, e sete dos 16 pacientes que referiram dor óssea faziam hemodiálise há mais de cinco anos. As demais alterações diretamente associadas ao tratamento dialítico prolongado como a STC, as deformidades ósseas e as tumorações articulares, entre outras, também apresentaram, em sua maioria, tempo de tratamento dialítico superior a cinco anos.

Um único paciente apresentou artrite simétrica de mãos e punhos, podendo ser considerado característico da AAD, mas seu tempo de tratamento foi de um ano e sete meses, não sendo compatível com os dados da literatura que afirmam que os pacientes que desenvolvem este tipo de alteração geralmente fazem HD por mais de cinco anos ${ }^{(34,35,39)}$.

Não foi objetivo do nosso estudo avaliar a influência dos tipos de membranas dialíticas utilizadas, uma vez que o mesmo tipo de membrana (polissulfona) foi utilizado por todos os pacientes.

Segundo Muñoz-gómez et al. ${ }^{(40)}$, Sethi et al. ${ }^{(32)}$ e Bar$\operatorname{din}^{(41)}$, o uso de membrana sintética está associado com menores níveis da proteína amilóide e conseqüente retardo no início da doença. Ao contrário, Kessler et al. ${ }^{(35)}$, em estudo multicêntrico com 171 pacientes em HD por mais de dez anos, e Gueerlings et al. ${ }^{(34)}$, em estudo de casocontrole retrospectivo com pacientes em HD há nove anos, não observaram diferença significativa na prevalência de sinais clínicos e/ou radiológicos de AAD, quando comparado o uso da membrana de poliacrilonitrila com a de cuprofane. Ambos encontraram uma correlação positiva entre complicações articulares e a idade.

Schiffl et al. ${ }^{(36)}$ realizaram estudo retrospectivo com 89 pacientes em HD por no mínimo dez anos com um só tipo de membrana e afirmaram que os principais determinantes de amiloidose osteoarticular nesses pacientes permanecem sendo o número de anos em HD e a idade do paciente no início do tratamento. No entanto, acreditam que o tipo de membrana (biológica) e os elementos participantes do método dialítico (contaminação bacteriana do dialisado, por exemplo) têm influência na prevalência das MME associadas com HD crônica, segundo o que foi observado em seus pacientes. 
A utilização de uma membrana com melhor biocompatibilidade e capacidade de ultrafiltração do que a de cuprofane, tipo de membrana mais associado com o desenvolvimento de MME de acordo com vários autores ${ }^{(32,36,40,41)}$, pode ter sido responsável pelo baixo porcentual de pacientes com sinais e/ou sintomas de STC (3 pacientes), tenossinovite dos tendões flexores dos dedos (1 paciente) e artrite simétrica de mãos e punhos (1 paciente), manifestações clínicas tipicamente observadas na AAD. No entanto, com exceção do paciente com artrite, o tempo de tratamento dialítico correlacionou-se positivamente com o desenvolvimento de MME nesses pacientes.

Com relação à $\mathrm{AAD}$, até o momento apenas o transplante renal pode lentificar ou reduzir à metade a progressão da artropatia amilóide. O transplante renal melhora os sintomas da artropatia dialítica rapidamente progressiva, e pode interromper a progressão da doença, provavelmente por causa do efeito dos imunossupressores administrados nesses pacientes e à parada de deposição de proteínas amilóides. Embora os pacientes recebam elevadas doses de imunossupressores pós-transplante, os sintomas não costumam recorrer depois que o rim transplantado apresenta boa função ${ }^{(32,42)}$.

O uso de corticosteróides nos pacientes transplantados costuma aliviar os sintomas, embora seu uso indiscriminado não seja recomendado ${ }^{(32)}$. Permanece incerto, porém,

\section{REFERÊNCIAS}

1. Caner JEZ, Decker JL: Recurrent acute (? Gouty) arthritis in chronic renal failure treated with periodic hemodialysis. Am J Med 36: 571 582, 1964.

2. Johnson C, Graham CB, Curtis FK: Roentgenographic manifestations of chronic renal disease treated by periodic hemodialysis. Am J Roentgenol Radium ther Nucl Med 101(4): 915-926, 1967.

3. Draibe SA: Diálise crônica. In: Prado FC, Ramos J, Vale JR (eds.): Atualização terapêutica: manual prático de diagnóstico e tratamento. $20^{a}$ ed, Rio de Janeiro: Artes Médicas, 2001. 758-760.

4. Brown EA, Gower PE: Joint problems in patients on maintenance hemodialysis. Clin Nephrol 18(5): 247-250, 1982.

5. Pohlmeier R, Viennken J: Phosphate removal and hemodialysis conditions. Kidney Int 59(Suppl. 78): S190-S194, 2001.

6. Massry S, Bluestone R, Klinenberg JR, Coburn JW: Abnormalities of the musculoskeletal system in hemodialysis patients. Sem Arthritis Rheum 4(4): 321-349, 1975.

7. Goodman WG, Duarte MEL: Aluminum: effects on bone and role in the pathogenesis of renal osteodystrophy. Min Eletrolyte Metab 17: 221-232, 1991

8. Carvalho AB, Lucca Jr. L, Kubrusly M, Jorgetti V: Fisiopatologia, clínica e tratamento da osteodistrofia renal. In: Riella MC (eds.): Princípios de nefrologia e distúrbios hidroeletrolíticos. $3^{\mathrm{a}}$ ed., Rio de Janeiro: Guanabara Koogan, 1996. 494-502. se ocorre regressão dos depósitos amilóides após o sucesso do transplante ${ }^{(42)}$.

A proporção de pacientes com MME foi divergente dos porcentuais descritos na literatura, provavelmente por causa das diferenças metodológicas, em especial a casuística, o tipo de membrana utilizado (biológicas, em sua maioria), duração do tratamento dialítico e métodos complementares de diagnóstico. Esses fatores podem ter contribuído para as dificuldades de comparação dos nossos resultados com os da literatura.

Entretanto, parece concordante que, em relação às manifestações articulares, o tempo de tratamento dialítico é a variável que mais se associa com o desenvolvimento das MME.

A partir desses dados, conclui-se que a proporção de MME nos nossos pacientes foi de 34\%; as manifestações articulares foram as mais observadas (72,7\% dos pacientes) e a artralgia foi a queixa isolada mais freqüentemente encontrada (46,57\% das MME). Neste estudo, com exceção do tempo de tratamento dialítico, as demais variáveis não mostraram associação com o desenvolvimento de sinais e/ ou sintomas musculoesqueléticos. Os pacientes que apresentaram MME foram aqueles que tiveram um maior tempo médio de tratamento dialítico (59,8 meses), comprovando uma associação entre o tempo de tratamento dialítico e o surgimento de MME nesses pacientes.

9. Goodman WG, Coburn JW, Slatopolsky E, Salusky IB: Renal osteodystrophy in adults and children. In: Farrus MJ (eds.): Primer on the metabolic bone diseases of mineral metabolism. $3^{\text {a }}$ ed. Philadelphia: Lippincott-Raven, 1996. 341-360.

10. Menerey K, Braunstein E, Brown M, Swartz R, Brown C, Fox IH: Musculoskeletal symptoms related to arthropathy in patients receiving dialysis. J Rheumatol 15 (12): 1848-1854, 1988.

11. Mirahmadi KS, Coburn JW, Bluestone R: Calcific Periarthritis and hemodialysis. JAMA 223(5): 548-549, 1973.

12. Goldfarb M: Infecção osteoarticular em pacientes hemodialisados. Arq Bras Med 68(4): 225-227, 1994.

13. Bardin T: Les complications rhumatologiques et l' amylose des dialysés. Rev Prat 40(7): 619-624, 1990.

14. Nguyen M: Dialysis and the elderly patient: questions and concerns. American Society of Consultant Pharmacists, pp. 1-7, may. 1998. Disponível em: <http://www.ascp.com/public/pubs/tcp/1998/ may/qandc.shtml>. Acesso em: 24 de outubro de 2001.

15. Sethi D, Maher ER, Cary NRB: Dialysis amyloid presenting as acute arthritis. Nephron 50: 73-74, 1988.

16. Naidich JB, Mossey RT, McHeffey-Atkinson B, et al: Spondyloarthropathy from long-term hemodialysis. Radiology 167: 761-764, 1988.

17. Lazarus JM, Denker BM, Owen Jr. WF: Hemodialysis. In Brener BM (eds.): The Kidney, $5^{a}$ ed. Philadelphia: Saunders Co., 1996. v. II, 2441-2467. 
18. Karakida O, Aoki J, Kanno Y, et al: Hemodialysis-related arthropathy: a prospective MR study with SE and GRE sequences. Acta Radiol 38: 158-164, 1997.

19. Kuntz D, Naveau B, Bardin T, Drueke T, Treves R, Dryll A: Destructive Spondylarthropathy in hemodialyzed patients: a new syndrome. Arthritis Rheum 27(4): 369-375, 1984.

20. Sebert JL, Fardellone P, Marie A, et al: Destructive spondylarthropathy in hemodialyzed patients: possible role of amyloidosis (letter). Arthritis Rheum 29: 301-303, 1986.

21. Naito M, Ogata K, Nakamoto M, Goya T, Sugioka Y: Destructive spondylo-arthropathy during long-term haemodialysis. J Bone Joint Surg 74-B (5): 686-690, 1992.

22. Appel H, Sieper J, Distler A, Braun J: Severe destructive beta 2microglobulin arthropathy after 28 years of hemodialysis. Arthritis Rheum 40(4): 709, 1997.

23. Wendling D, Guidet M: Manifestations articulaires et périarticulaires de l' hémodialyse chronique chez l'adulte. Revue du Rhumatisme 53(12): 715-21, 1986.

24. Netter P, Kessler M, Burnel D, et al: Aluminum in the joint tissues of chronic renal failure patients treated with regular hemodialysis and aluminum compounds. J Rheumatol 11(1): 66-70, 1984.

25. Chattopadhyay C, Ackrill P, Clague RB: The shouder pain syndrome and soft-tissue abnormalities in patients on long-term haemodialysis. Br J Rheumatol 26(3): 181-187, 1987.

26. Ferrari AJ, Rothfuss S, Schumacher HRJ: Dialysis arthropathy: identification and evaluation of a subset of patients with unexplained inflammatory effusions. J Rheumatol 24(9): 1780-1786, 1997.

27. Bailey GL, Griffiths HJL, Mocelin AJ, et al: A vascular necrosis of the femoral head in patients on chronic hemodialysis. Am Soc Artif Intern Organs 18: 401-4, 1972.

28. Goldstein S, Winston E, Chung TJ, Chopra S, Pariser K: Chronic arthropathy in long-term hemodialysis. Am J Med 78: 82-86, 1985.

29. Hardouin P, Flipo R-M, Foissac-Gegoux P, et al: Current aspects of osteoarticular pathology in patients undergoing hemodialysis: study of 80 patients. J Rheumatol 14(4): 780-783, 1987.

30. Chou CT, Wasserstein A, Schumacher Jr. HR, Fernadez P: Musculoskeletal manifestations in hemodialysis patients. J Rheumatol 12(6): 1149-53, 1985.
31. Van ypersele de Strihou C, Jadoul M, Malghem J, Maldague B, Jamart J: Effect of dialysis membrane and patient's age on signs of dialysis-related amyloidosis. Kidney Int 39: 1012-1019, 1991.

32. Sethi D, Naunton Morgan TC, Brown EA, et al: Dialysis arthropathy: a clinical, biochemical, radiological and histological study of 36 patients. Quart J Med 77(282): 1061-1082, 1990.

33. Gaucher A, Kessler M, Netter P, Azoulay E, Pere P, Mur J-M: Dialysis arthrophaty: the effect of age. J Rheumatol 15: 1880-1, 1988.

34. Gueerlings W, Rizzoni G, Selwood NH, et al: Case control study on dialysis arthropathy: the influence of two different dialysis membranes: data from the European Dialysis Transplant Association registry. Nephrol Dial Transplant 5: 432-436, 1990.

35. Kessler M, Netter P, Azoulay E, Mayeux D, Pere P, Gaucher A: Dialysis-associated arthropathy: a multicenter survey of 171 patients receiving haemodialysis for over 10 years. Br J Rheumatol 31: 157162, 1992.

36. Schiffl H, Fischer R, Lang SM, Mangel E: Clinical manifestations of AB-amyloidosis: effects of biocompatibility and flux. Nephrol Dial Transplant 15: 840-845, 2000.

37. Rubin LA, Fam AG, Rubenstein J, Campbel J, Saiphoo C: Erosive azotemic osteoarthropathy. Arthritis Rheum 27(10): 1086-1094, 1984.

38. Halter SK, DeLisa JÁ, Stolov WC: Carpal tunnel syndrome in chronic renal dialysis patients. Arch Phys Med Rehabil 62: 197201, 1981.

39. Nangaku M, Miyata T, Kurokawa K: Pathogenesis and management of dialysis-related amyloid bone disease. Am J Med Sciences 317(6): 410-415, 1999.

40. Muñoz-gómez J, Gómez-perez R, Llopart-buisán E, Solé-arqués M: Clinical picture of the amyloid arthropathy in patients with chronic renal failure maintained on haemodialysis using cellulose membranes. Ann Rheum Dis 46: 573-579, 1987.

41. Bardin T: Muskuloskeletal problems in dialysis patients. In Klippel $\mathrm{JH}$ (eds.): Primer on the Rheumatic disease. $11^{\text {th }}$ ed. Atlanta: Arthritis Foundation, 1997. 356-357.

42. Miyata T, Inagi R, Kurokawa K: Diagnosis, pathogenesis, and treatment of dialysis-related amyloidosis. Miner Electrolyte Metab 25: 114-117, 1999. 